УДК 517.1

https://doi.org/10.36906/AP-2020/53

\title{
РАСЧЕТ ПАРАМЕТРОВ ЭКСТРЕМАЛЬНОГО КОМПЛЕКСНОЗНАЧНОГО СПЛАЙНА В ЗАДАЧЕ О БЫСТРОДЕЙСТВИИ ФУНКЦИЙ ЧЕРЕЗ ВНУТРЕННИЙ И ВНЕШНИЙ РАДИУСЫ КОЛЬЦА ЕЕ ЗНАЧЕНИЙ
}

\author{
Дмитриев Н. П. \\ канд. пед. наук \\ Нижневартовский государственный университет \\ 2. Нижневартовск, Россия \\ Юмагулов Н. И. \\ канд. пед. наук \\ Нижневартовский государственный университет \\ г. Нижневартовск, Россия
}

Аннотация. В задаче о быстродействии комплекснозначных функций используется экстремальный параболический периодический по аргументу сплайн, с помощью которого были найдены соответствующие оценки быстродействия. В настоящей заметке рассчитываются параметры такого сплайна через внутренний и внешний радиусы кольца ее значений, необходимые для моделирования оптимальной траектории движения.

Ключевые слова: неравенство Адамара; сплайны Эйлера; оценки нормы производной; контактные точки; оценки быстродействия комплекснозначных функций.

На классе $\bar{W}^{2}$ заданных на всей числовой прямой $R$ комплекснозначных дифференцируемых функций $f(t)$ действительного аргумента $t$ с абсолютно непрерывной производной $f^{\prime}(t)$ на любом отрезке из $R$ с чебышевскими нормами функции и ее производных

$$
\begin{gathered}
\|f\|=\sup _{t}|f(t)|=\sup _{t}\left(\operatorname{Re}^{2} f(t)+\operatorname{Im}^{2} f(t)\right)^{1 / 2}, \\
\left\|f^{\prime}\right\|=\sup _{t}\left|f^{\prime}(t)\right|=\sup _{t}\left(\operatorname{Re}^{2} f^{\prime}(t)+\operatorname{Im}^{2} f^{\prime}(t)\right)^{1 / 2}, \\
\left\|f^{\prime \prime}\right\|=\operatorname{ess} \sup _{t}\left|f^{\prime \prime}(t)\right|=\operatorname{ess} \sup _{t}\left(\operatorname{Re}^{2} f^{\prime \prime}(t)+\operatorname{Im}^{2} f^{\prime \prime}(t)\right)^{1 / 2}
\end{gathered}
$$

рассчитаем параметры экстремального сплайна [1] через внутренний и внешний радиусы кольца значений функций $f(t)$.

Изучение свойств такого сплайна тесно связано с задачами оценки норм производных с ограничениями на норму функции и ее производной второго порядка. Так Ж. Адамар [2] получил точную оценку нормы производной действительной функции. А именно, пусть. Тогда верно, точное неравенство: $\left\|f^{\prime}\right\| \leq \sqrt{2 M_{0} M_{2}}$. И. Шенберг [3] получил точную оценку нормы производной комплекснозначной функции. А именно, пусть $N_{0} \leq\|f\| \leq M_{0},\left\|f f^{\prime \prime}\right\| \leq 1$. Тогда верно, точное неравенство: $\left\|f^{\prime}\right\| \leq \sqrt{M_{0}-\sqrt{M_{0}^{2}-N_{0}^{2}}}$. Точные оценки, как правило, реализуются на специально построенных экстремальных функциях. 
По определению комплекснозначный сплайн $g_{r}(t, v)(r=0,1,2, \ldots)$ в случае $r=2$ имеет следующее каноническое представление [1]:

$$
g_{2}(t, v)=\left(f_{2}(t)-\mu_{2}(v)\right)+i \mu_{1}(v) f_{1}(t)
$$

где $f_{1}(t), f_{2}(t)$ - идеальные сплайны Эйлера, $i=\sqrt{-1}, \quad 0 \leq v \leq \frac{\pi}{2}, \quad-\frac{\pi}{2} \leq t \leq \frac{\pi}{2}$, $\mu_{1}(v)=K_{1} \operatorname{ctg} v, \mu_{2}(v)=K_{1}^{2} \operatorname{ctg}^{2} v$.

В [1] контактные точки области изменения функции $f \in \bar{W}^{r}$ с ограничением $\|f\| \leq M_{0}$ - это набор $D=\left\{D_{1}, D_{2}, \ldots, D_{p}\right\}$ точек $D_{1}, D_{2}, \ldots, D_{p}$ комплексной плоскости $C$ таких, что

$$
\begin{aligned}
& \left|D_{1}\right|=\left|D_{2}\right|=\ldots=\left|D_{p}\right|=M_{0} \\
& D_{j+1}=D_{j} e^{2 \pi i / p} \quad(j=1,2, \ldots, p-1) \quad D_{1}=D_{p} e^{2 \pi i / p}
\end{aligned}
$$

где $i=\sqrt{-1}$ - мнимая единица. Другими словами, контактные точки - точки, равномерно расположенные на окружности $\|f\|=M_{0}$. Порядок обхода контактных точек обозначим последовательностью их индексов. Ниже на рисунке 1 представлен сплайн в случае $p=5$ с порядком обхода $Q=\{1,3,5,2,4,1\}$.

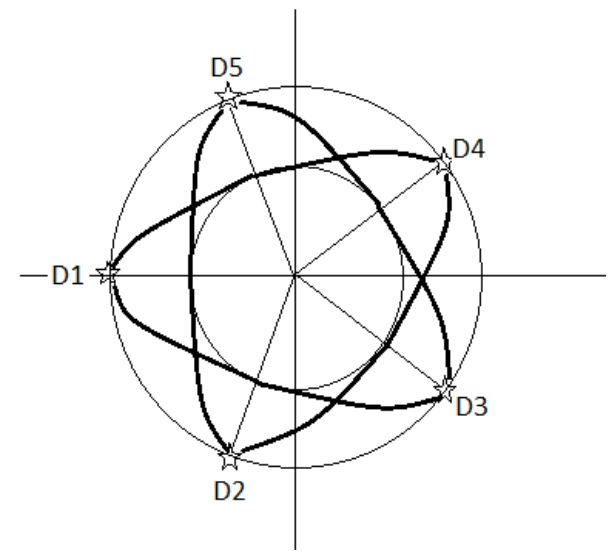

Рис. 1. Экстремальный комплекснозначный сплайн $g_{2}(t, v)$

Подставляя явные выражения функций, $f_{2}(t)$ в (1), получаем

$$
g_{2}(t, v)=\left(\frac{t^{2}}{2}-\frac{\pi^{2}}{8} \frac{1+\cos ^{2} v}{\sin ^{2} v}\right)+i \frac{\pi}{2} \frac{\cos v}{\sin v} t
$$

Найдем экстремальные значения модуля производной этого сплайна. Наибольшее значение модуль производной сплайна $g_{2}(t, v)$ достигает в точке $t=0$, а наименьшее — в точке $t=\frac{\pi}{2}$. Таким образом,

$$
\begin{aligned}
& \left\|g_{2}(\cdot, v)\right\|=\left|g_{2}(0, v)\right|=\frac{\pi^{2}}{8} \frac{1+\cos ^{2} v}{\sin ^{2} v} \\
& \forall g_{2}(\cdot, v) \diamond=\left|g_{2}\left(\frac{\pi}{2}, v\right)\right|=\left(\left(\frac{\pi^{2}}{8}-\frac{\pi^{2}}{8} \frac{1+\cos ^{2} v}{\sin ^{2} v}\right)^{2}+\left(\frac{\pi}{2} \frac{\cos v}{\sin v} \frac{\pi}{2}\right)^{2}\right)^{1 / 2}= \\
& =\left(\frac{\pi^{4}}{64} \frac{4 \cos ^{4} v}{\sin ^{4} v}+\frac{\pi^{4}}{16} \frac{\cos ^{2} v}{\sin ^{2} v}\right)^{1 / 2}=\frac{\pi^{2}}{4} \frac{\cos v}{\sin ^{2} v}
\end{aligned}
$$


Отсюда получаем

$$
\begin{gathered}
K_{2}(v)=\left\|g_{2}(\cdot, v)\right\|=K_{2} \frac{1+\cos ^{2} v}{\sin ^{2} v} \\
L_{2}(v)=\diamond g_{2}(\cdot, v) \oslash=K_{2} \frac{2 \cos v}{\sin ^{2} v}
\end{gathered}
$$

Пусть $M_{0}$ и $N_{0}\left(0 \leq N_{0} \leq M_{0}\right)$ означают внешний и внутренний радиусы кольца значений функции $f \in \bar{W}^{r}$. Найдем параметры $\lambda, v$, при которых имеют место равенства

$$
\begin{gathered}
\left\|g_{\lambda, 2}(\cdot, v)\right\|=M_{0} \\
\diamond g_{\lambda, 2}(\cdot, v) \oslash=N_{0}
\end{gathered}
$$

Положим

$$
g_{\lambda, 2}(t, v)=\frac{1}{\lambda^{2}} g_{2}(\lambda t, v)
$$

Тогда

$$
\begin{aligned}
\left\|g_{\lambda, 2}(\cdot, v)\right\| & =\frac{1}{\lambda^{2}}\left\|g_{2}(\cdot, v)\right\| \\
\diamond g_{\lambda, 2}(\cdot, v) \diamond & =\frac{1}{\lambda^{2}} \oslash g_{2}(\cdot, v) \diamond
\end{aligned}
$$

Подставляя (8) в (5), а (9) в (6), получаем

$$
\frac{1}{\lambda^{2}}\left\|g_{2}(\cdot, v)\right\|=M_{0} \quad \frac{1}{\lambda^{2}} \diamond g_{2}(\cdot, v) \diamond=N_{0}
$$

С учетом (3), (4) выводим

$$
\begin{gathered}
\frac{1}{\lambda^{2}} K_{2} \frac{1+\cos ^{2} v}{\sin ^{2} v}=M_{0} \\
\frac{1}{\lambda^{2}} K_{2} \frac{2 \cos v}{\sin ^{2} v}=N_{0}
\end{gathered}
$$

Исключая $\lambda$ из равенств (10), (11), приходим к следующему уравнению относительно $\cos v:$

$$
\cos ^{2} v-\frac{2 M_{0}}{N_{0}} \cos v+1=0
$$

Отсюда имеем:

$$
\cos v=\frac{M_{0}-\sqrt{M_{0}^{2}-N_{0}^{2}}}{N_{0}}
$$

С помощью равенств (10) или (11) получаем

$$
\begin{gathered}
\lambda=\sqrt{\frac{K_{2}}{M_{0}}} \frac{\sqrt{1+\cos ^{2} v}}{\sin v} \\
\lambda=\sqrt{\frac{K_{2}}{N_{0_{2}}}} \sqrt{\frac{2 \cos v}{\sin ^{2} v}}
\end{gathered}
$$


Таким образом, если параметры $\lambda, v$ выбирать по формулам (12), (13), (14), то график значений параболического сплайна $g_{\lambda, 2}(t, v)$ окажется вписанным в центральное кольцо $C\left(M_{0}, N_{0}\right)$ комплексной плоскости с внешним радиусом $M_{0}$ и внутренним радиусом $N_{0}$.

Выразим константы $K_{2}(v)$ и $L_{2}(v)$ через заданные внешний $M_{0}$ и внутренний $N_{0}$ радиусы кольца $C\left(M_{0}, N_{0}\right)$ :

$$
\begin{aligned}
& K_{2}(v)=K_{2} \frac{M_{0}}{\sqrt{M_{0}^{2}-N_{0}^{2}}} \\
& L_{2}(v)=K_{2} \frac{N_{0}}{\sqrt{M_{0}-N_{0}}}
\end{aligned}
$$

Ясно, что если $N_{0} \rightarrow M_{0}$, то $K_{2}(v) \rightarrow \infty$ и $L_{2}(v) \rightarrow \infty$. Если же $N_{0}=0$, то $K_{2}(v)=K_{2}$, $L_{2}(v)=0$.

По определению параметр $v$ меняется на промежутке $\left[0, \frac{\pi}{2}\right]$, тогда $0 \leq \cos v \leq 1$. Эти же ограничения вытекают из (12) при изменении внутреннего радиуса кольца $C\left(M_{0}, N_{0}\right)$.

Очевидно, что в качестве управляющего параметра при изучении параболического сплайна $g_{\lambda, 2}(\cdot, v)$ можно вместо параметра $v$ взять внутренний радиус кольца значений этого сплайна при условии, что внешний радиус зафиксирован. В этом случае получаем следующее представление сплайна $g_{\lambda, 2}(\cdot, v)$ и его производной:

$$
\begin{gathered}
\bar{g}_{2}\left(t, N_{0}\right)=\left(\frac{t^{2}}{2}-M_{0}\right)+i \sqrt{M_{0}-\sqrt{M_{0}^{2}-N_{0}^{2}}} t \\
\bar{g}_{1}\left(t, N_{0}\right)=t+i \sqrt{M_{0}-\sqrt{M_{0}^{2}-N_{0}^{2}}} \\
0<t<\sqrt{2} \sqrt[4]{M_{0}^{2}-N_{0}^{2}}
\end{gathered}
$$

Правая граница промежутка изменения аргумента $t$ найдена с помощью формул (15), (16). Отсюда имеем:

$$
\begin{aligned}
\left\|\bar{g}_{1}\left(t, N_{0}\right)\right\| & =\sqrt{M_{0}+\sqrt{M_{0}^{2}-N_{0}^{2}}} \\
\diamond \bar{g}_{1}\left(t, N_{0}\right) \diamond & =\sqrt{M_{0}-\sqrt{M_{0}^{2}-N_{0}^{2}}}
\end{aligned}
$$

Заметим, что при фиксированном внешнем радиусе $M_{0}$ кольца $C\left(M_{0}, N_{0}\right)$ значений параболического комплекснозначного сплайна величина $\left\|\overline{g_{1}}\left(\cdot, N_{0}\right)\right\|$ монотонно убывает от $\sqrt{2 M_{0}}$ до $\sqrt{M_{0}}$, а величина $\diamond \bar{g}_{1}(\cdot, v) \diamond$ возрастает от 0 до $\sqrt{M_{0}}$.

Формулы (19) и (20), в частности, указывают на то, что максимальная скорость движущегося по траектории $g_{\lambda, 2}(t, v)$ объекта достигается в точке касания с внутренней границей кольца $C\left(M_{0}, N_{0}\right)$, а минимальная скорость - в точке касания с внешней границей кольца значений $f \in \bar{W}^{r}$. 


\section{СОВРЕМЕННОЕ ПРОГРАММИРОВАНИЕ}

III Международная научно-практическая конференция

\section{Литература}

1. Дмитриев Н. П. Теоремы сравнения и оценки быстродействия комплекснозначных функций с ограничениями // Lap Lambert (Германия, Saarbrucken), 2014. 75 c.

2. Hadamard J. Sur le module maximum d'une function et de ses derives // Soc. Math. France. Comptes rendus des Seanses. 914. 41. P. 68-72.

3. Schoenberg I. J. The Landau problem I. The case of motion on sets // Proc. Scand. Acad. Sci. 1978. Vol. 81. №2. P. 218-231.

СДдитриев Н. П., Юмагулов Н. И., 2020 